\title{
ODONTOLOGIA E GESTAÇÃO: A IMPORTÂNCIA DO PRÉ-NATAL ODONTOLÓGICO
}

DENTISTRY AND PREGNANCY: THE IMPORTANCE OF DENTAL PRENATAL CARE

ODONTOLOGÍA Y EMBARAZO: LA IMPORTANCIA DE LA ATENCIÓN PRENATAL ODONTOLÓGICA

Diana Larissa Leitão Botelho ${ }^{1}$

Vivian Gomes Azevedo Lima 2

Myrna Maria Arcanjo Frota Barros 3

Janaina Rocha de Sousa Almeida ${ }^{4}$

Palavras-chave:

Odontologia; Sistema Único de Saúde; Estratégia Saúde da Família; Gestação.

Keywords:

Dentistry; Brazilian National Health System; Family Health Strategy; Pregnancy.

Palabras clave: Odontología; Sistema Nacional de Salud de Brasil; Estrategia Salud de la Familia; Embarazo.

Submetido: $16 / 07 / 2019$

Aprovado: $10 / 12 / 2019$

Autor(a) para Correspondência: Myrna Maria Arcanjo Frota Barros

R. Conselheiro José Júlio, $S / N$ Centro - Sobral (CE) 62010-820

E-mail:myrnaarcanjo@ufc.br

\section{RESUMO}

o objetivo deste estudo foi conhecer a percepção das gestantes sobre a assistência odontológica no pré-natal. Realizado em Fortaleza (CE), no período de agosto de 2015 a julho de 2016, nas unidades de atenção primária à saúde (UAPS) da Secretaria Regional II. Dados quantitativos foram coletados por meio de questionário semiestruturado, com dados sociodemográficos e odontológicos. As informações qualitativas foram coletadas mediante entrevistas, nas quais se estabeleceu uma situação de conversa informal, guiada por temas que aprofundaram as opiniões dos participantes. As gestantes reconhecem a importância do tratamento odontológico no período pré-natal, porém, o acompanhamento com o cirurgião-dentista ainda não é uma rotina nos serviços de saúde em análise.

\footnotetext{
1. Aluna de Graduação em Odontologia na Universidade Federal do Ceará (UFC). E-mail: ladianabotelho@gmail. com ORCID: https://orcid.org/0000-0001-5963-118X

2. Aluna de Graduação em Odontologia no Centro Universitário Fametro (UniFametro). E-mail: viviangomes_ azevedolima@hotmail.com ORCID: https://orcid.org/0000-0001-9930-7595

3. Cirurgiã-Dentista. Doutora em Clínica 0dontológica pela UFC. E-mail: myrnaarcanjo@ufc,br ORCID: https:// orcid.org/0000-0001-7689-175X

4. Cirurgiã-Dentista. Doutora em Clínica Odontológica pela UFC. Professora no Centro Universitário Christus (UniChristus). E-mail; drajanainarocha@hotmail.com ORCID: https://orcid.org/0000-0002-6736-3936
} 


\section{ABSTRACT}

This study aimed to know pregnant women's perception of prenatal dental care. It was conducted in Fortaleza, Ceará, Brazil, within the period from August 2015 to July 2016, at the primary health care centers (unidades de atenção primária à saúde [UAPS]) of the Regional Department II. Quantitative data were collected by means of a semi-structured questionnaire, with sociodemographic and dental data. Qualitative information was collected through interviews, in which a situation of informal conversation was established, guided by themes that provided in-depth participants' opinions. Pregnant women recognize the importance of dental treatment within the prenatal period, but follow-up with a dental surgeon is not a routine in the health services under analysis, yet.

\section{RESUMEN}

Este estudio tuvo como objetivo conocer la percepción de mujeres embarazadas acerca de la atención prenatal odontológica. Realizado en Fortaleza, Ceará, Brasil, dentro del período comprendido entre agosto de 2015 y julio de 2016, en las unidades de atención primaria de salud (unidades de atenção primária à saúde [UAPS]) de la Secretaría Regional II. Datos cuantitativos se recopilaron mediante un cuestionario semi-estructurado, con datos sociodemográficos y dentales. La información cualitativa se recopiló a través de entrevistas, en las que se estableció una situación de conversación informal, guiada por temas que proporcionaron opiniones profundas de los participantes. Las mujeres embarazadas reconocen la importancia del tratamiento dental dentro del periodo prenatal, pero el seguimiento con un cirujano dental aún no es una rutina en los servicios de salud bajo análisis.

\section{INTRODUÇÃ O}

A gestação é um evento fisiológico, com alterações orgânicas naturais, que impõe aos profissionais da saúde a necessidade de adquirir conhecimentos específicos para uma assistência humanizada e integral. Nesse momento único na vida da mulher, ela se encontra mais receptiva a novos conhecimentos relacionados à sua saúde e à saúde de seu bebê, portanto, mostra-se essencial a atuação dos serviços sob a perspectiva de promoção da saúde, educação em saúde e prevenção de agravos ${ }^{1-3}$.

Nesse sentido, destaca-se que a mulher assume papel-chave dentro da família, tornando-se multiplicadora de informações sobre os cuidados com a saúde de todo o núcleo familiar. Portanto, devese enfatizar as atividades de educação em saúde, com vistas à promoção da saúde tanto da futura mãe quanto do nascituro ${ }^{4}$.

Contudo, muitas vezes, o estado de saúde bucal é negligenciado durante a gestação. As evidências científicas comprovam a correlação entre doenças da cavidade bucal e o período gestacional ${ }^{1-2,5-11}$. Por isso, as equipes de saúde bucal devem trabalhar de modo integrado com os demais profissionais da equipe de saúde e, no que diz respeito à gestante, trabalhase em constante interação com os profissionais responsáveis por seu atendimento ${ }^{10}$.

Orientações do Ministério da Saúde (MS) recomendam que a gestante, ao iniciar o prénatal, deve ser encaminhada para uma consulta odontológica, durante a qual receberá orientações sobre a possibilidade de atendimento durante a gestação, exames de tecidos moles, identificação de risco à saúde bucal, diagnóstico de lesões de cárie, necessidade de tratamento curativo, diagnóstico de gengivite ou doença periodontal crônica, necessidade de tratamento e orientações sobre hábitos alimentares e higiene bucal ${ }^{10}$.

Uma dúvida frequente entre os cirurgiõesdentistas é:

- Qual é o período mais adequado para o atendimento de gestantes?

Ressalta-se que o primeiro trimestre se mostra - período menos adequado para o tratamento odontológico, pois nele ocorrem as principais transformações embriológicas. Além disso, a maioria das pacientes pode apresentar indisposição, enjoos matutinos e náuseas à menor provocação. Nesse período, devem-se evitar, principalmente, tomadas radiográficas. 0 segundo trimestre é o período mais adequado para intervenções clínicas e procedimentos odontológicos essenciais, sempre de acordo com as indicações. Durante esse período, a organogênese está completa e o feto já está desenvolvido. A mãe se sente mais confortável do que nos estágios iniciais ou finais de sua gravidez. Existe apenas o período de hipotensão postural, se a paciente for tratada na posição supina e houver uma mudança brusca para a posição em pé. 0 terceiro trimestre é um momento de maior risco de síncope, hipertensão e anemia. 0 desconforto na cadeira odontológica é 
frequente, podendo ocorrer hipotensão postural. É prudente evitar um tratamento odontológico nesse período ${ }^{11-13}$.

Portanto, a saúde bucal da gestante ainda é cercada de muitas dúvidas e crenças passadas ao longo dos tempos por meio de experiências relatadas por familiares e outras gestantes, já que as gestantes desconhecem fatores biomédicos importantes referentes à saúde bucal, não colocando o pré-natal odontológico como prioridade, o que contribui para o agravo de problemas bucais e para a precariedade das condições bucais tanto das mães quanto de seus filhos ${ }^{2}$

Com isso, torna-se indispensável que as equipes de saúde invistam na perspectiva de desmitificar crenças sobre os cuidados de saúde bucal durante a gestação, respeitando os saberes populares, contudo, informando e orientando as gestantes sobre o saber científico, de modo a destacar a importância da prevenção de agravos e promoção da saúde durante o pré-natal².

É notório que, durante o período gestacional, a mulher aumenta seus cuidados gerais de saúde, sendo importante não esquecer a saúde bucal. Porém, algumas gestantes ainda acreditam em mitos e crenças populares relativos ao tratamento odontológico. Portanto, a desmitificação desses fatores é importante para a execução de um tratamento odontológico de qualidade no período do pré-natal ${ }^{6}$.

Diante do exposto, o objetivo deste estudo foi:

- Conhecer a percepção das gestantes sobre a assistência odontológica no pré-natal.

\section{METODOLOGIA}

0 estudo se caracteriza como qualiquantitativo, realizado em Fortaleza (CE). A coleta de dados ocorreu no período de agosto de 2015 a julho de 2016, nas unidades de atenção primária à saúde (UAPS) da Secretaria Regional II. A capital cearense é dividida em seis Secretarias Regionais, que são agrupadas de acordo com as características da população e do território. A população em questão consistiu nas gestantes que realizavam pré-natal nas UAPS indicadas.

Os dados quantitativos foram coletados por meio de questionário semiestruturado, composto por 3 partes: a) dados pessoais e socioeconômicos (idade, escolaridade, estado civil, ocupação); b) dados sobre a gestação (tempo da gestação e número da

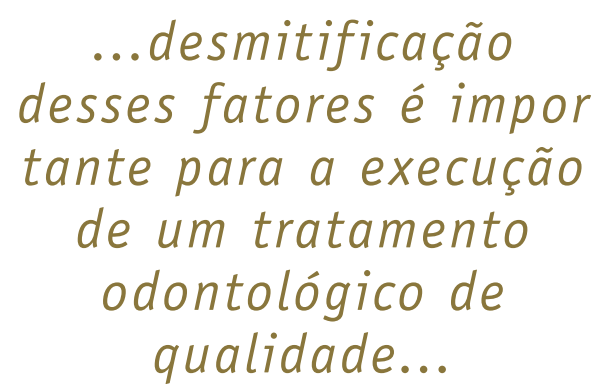

gestação); e c) dados odontológicos (hábitos de higiene bucal, mitos e crenças sobre o tratamento odontológico na gestação). 0 questionário foi validado por especialistas no assunto e, em seguida, foi submetido a um estudo piloto conduzido com um grupo de gestantes de uma UAPS que não faria parte da amostra, visando à adequação do instrumento aos objetivos propostos. As participantes foram 61 gestantes acompanhadas pelas UAPS durante a execução da pesquisa.

Os dados foram tabulados no software Microsoft Excel e exportados para o software SPSS, versão 17.0 para Microsoft Windows, no qual as análises descritivas e inferenciais foram processadas ( $p$ $<0.05)$. Os dados quantitativos foram expressos em forma de média e desvio padrão e os dados categóricos foram expressos em forma de frequência absoluta e percentual e submetidos a teste quiquadrado de Pearson ou teste exato de Fisher.

Todas as gestantes confirmaram seu consentimento em participar na pesquisa via assinatura do termo de consentimento livre e esclarecido (TCLE). A abordagem qualitativa utilizou a técnica de entrevista com roteiro previamente estabelecido para coleta de dados, na qual se estabelece uma situação de conversa informal, guiada por temas, que aprofunda as opiniões dos sujeitos de pesquisa. 0 ambiente se caracterizou como neutro e propiciou conforto às participantes para a exposição de suas opiniões.

Os dados qualitativos foram categorizados, verificando a frequência da repetição de opiniões e termos. As entrevistas foram transcritas e lidas na íntegra, a fim de categorizar as falas. Todas as entrevistas foram codificadas, categorizadas e sistematizadas por meio de análise compreensiva. Adotaram-se nomes fictícios para preservar a identidade das entrevistadas.

0 estudo foi submetido ao Comitê de Ética

e Pesquisa do Centro Universitário Christus (UniChristus), em conformidade com a Resolução n. 
466/2012, do Conselho Nacional de Saúde (CNS), sendo aprovado sob o Parecer n. 1.068.366/2014.

\section{RESULTADOS}

A idade média das gestantes foi $25,1 \pm 6,7$ anos. A amostra foi composta por 61 gestantes; $42,6 \%$ reportaram ter Ensino Médio completo, 60,7\% eram solteiras, 49,2\% eram donas de casa e 37,7\% se encontravam na segunda gravidez, como ilustra a Tabela 1.

Tabela 1 - Dados sociodemográficos e odontológicos de gestantes. Fortaleza, 2015-2016.

\begin{tabular}{|c|c|c|}
\hline & $\mathbf{N}$ & $\%$ \\
\hline \multicolumn{3}{|l|}{ Escolaridade } \\
\hline Ensino Fundamental incompleto & 20 & 32,8 \\
\hline Ensino Fundamental completo & 6 & 9,8 \\
\hline Ensino Médio incompleto & 7 & 11,5 \\
\hline Ensino Médio completo & 26 & 42,6 \\
\hline Ensino Superior & 1 & 1,6 \\
\hline Nunca estudou & 1 & 1,6 \\
\hline \multicolumn{3}{|l|}{ Estado civil } \\
\hline Casada & 20 & 32,8 \\
\hline Solteira & 37 & 60,7 \\
\hline Separada & 1 & 1,6 \\
\hline Outros & 3 & 4,9 \\
\hline \multicolumn{3}{|l|}{ Ocupação } \\
\hline Trabalha fora & 20 & 32,8 \\
\hline Dona de casa & 30 & 49,2 \\
\hline Estudante & 9 & 14,8 \\
\hline Outras & 2 & 3,3 \\
\hline \multicolumn{3}{|l|}{ Número de gestações } \\
\hline Uma & 20 & 32,8 \\
\hline Duas & 23 & 37,7 \\
\hline Três & 10 & 16,4 \\
\hline Quatro & 5 & 8,2 \\
\hline Outras & 3 & 4,9 \\
\hline \multicolumn{3}{|c|}{ Última visita ao cirurgião-dentista } \\
\hline Menos de 6 meses & 38 & 62,3 \\
\hline 1 ano & 7 & 11,5 \\
\hline Mais de 1 ano & 2 & 3,3 \\
\hline 2 anos & 7 & 11,5 \\
\hline Outras & 7 & 11,5 \\
\hline \multicolumn{3}{|c|}{ Autopercepção da necessidade de tratamento odontológico } \\
\hline Sim & 57 & 93,4 \\
\hline Não & 2 & 3,3 \\
\hline Não sei & 2 & 3,3 \\
\hline Total & 61 & 100,0 \\
\hline \multicolumn{3}{|c|}{ Realiza acompanhamento com o cirurgião-dentista } \\
\hline Sim & 26 & 42,6 \\
\hline Não & 35 & 57,4 \\
\hline
\end{tabular}




\begin{tabular}{|c|c|c|}
\hline & $\mathbf{N}$ & $\%$ \\
\hline \multicolumn{3}{|c|}{ Realiza escovação dental } \\
\hline Sim & 61 & 100,0 \\
\hline \multicolumn{3}{|c|}{ Utiliza fio dental } \\
\hline Sim & 26 & 43,3 \\
\hline Não & 26 & 43,3 \\
\hline Às vezes & 8 & 13,3 \\
\hline Total & 60 & 100,0 \\
\hline \multicolumn{3}{|c|}{ Ocorre alteração na saúde bucal durante a gestação } \\
\hline Sim & 18 & 30,9 \\
\hline Não & 42 & 70,0 \\
\hline Não sei & 1 & 1,6 \\
\hline \multicolumn{3}{|c|}{ Foi orientada sobre a saúde bucal } \\
\hline Sim & 32 & 52,5 \\
\hline Não & 28 & 45,9 \\
\hline Não sei & 1 & 1,6 \\
\hline Total & 61 & 100,0 \\
\hline \multicolumn{3}{|c|}{ Existe procedimento odontológico que não pode ser realizado durante a gestação } \\
\hline Sim & 21 & 34,4 \\
\hline Não & 30 & 49,2 \\
\hline Não sei & 10 & 16,4 \\
\hline \multicolumn{3}{|c|}{ Procedimentos que não podem ser realizados durante a gestação } \\
\hline Anestesia & 10 & 16,4 \\
\hline Raio-X & 8 & 13,1 \\
\hline Exodontia & 11 & 18,0 \\
\hline Endodontia & 7 & 11,7 \\
\hline Restauração & 3 & 4,9 \\
\hline \multicolumn{3}{|c|}{ Influência da saúde bucal na gestação } \\
\hline Sim & 44 & 72,1 \\
\hline Não & 13 & 21,3 \\
\hline Não sei & 4 & 6,6 \\
\hline
\end{tabular}

Fonte: Elaborada pelas autoras.

Ao avaliar o acesso ao serviço odontológico, verifica-se que $62,3 \%$ das gestantes foram a consultório odontológico havia menos de 6 meses. Quanto à autopercepção sobre sua necessidade de tratamento odontológico, 93,4\% compreendiam que tinham necessidade de tratamento odontológico, porém 57,4\% não realizavam acompanhamento com o cirurgião-dentista durante o pré-natal.

Constatou-se que $100 \%$ das participantes realizam sua higiene bucal normalmente e 43,3\% relatam o uso de fio dental. Para $70 \%$ das gestantes não ocorreram alterações em sua saúde bucal durante a gestação e $52,5 \%$ relatam ter sido orientadas sobre sua saúde bucal.

Apesar dos mitos relacionados a atendimento odontológico no período gestacional, 49,2\% das gestantes acreditam não existir nenhum procedimento odontológico que não possa ser realizado durante o pré-natal. Já $16,4 \%$ das gestantes não teriam permitido o uso de anestesia durante a gravidez, os exames radiográficos devem ser proibidos em gestantes de acordo com 13,1\% das entrevistadas, a realização de exodontias deve ser evitada para $18 \%$ delas e $11,7 \%$ acreditam que o tratamento endodôntico não deve ser realizado durante a gravidez. Além disso, os tratamentos restauradores são considerados procedimentos menos invasivos, por isso apenas $4,9 \%$ acreditam que não devem ser realizados no período gestacional. A influência da saúde bucal na gestação foi considerada evidente por $72,1 \%$ das gestantes.

A Tabela 2 apresenta a correlação entre a escolaridade das gestantes e as variáveis autopercepção sobre a 
necessidade de tratamento odontológico, acompanhamento com o cirurgião-dentista, recebimento de orientação sobre a saúde bucal e procedimentos odontológicos que não podem ser realizados durante a gestação.

Tabela 2 - Avaliação da percepção das gestantes sobre a saúde bucal de acordo com a escolaridade. Fortaleza, 2015-2016.

\begin{tabular}{|c|c|c|c|c|c|c|c|}
\hline & \multicolumn{6}{|c|}{ Escolaridade } & \multirow[b]{2}{*}{ Valor $p$} \\
\hline & $\begin{array}{c}\text { Ensino } \\
\text { Fundamental } \\
\text { incompleto }\end{array}$ & $\begin{array}{c}\text { Ensino } \\
\text { Fundamental } \\
\text { completo }\end{array}$ & $\begin{array}{c}\text { Ensino } \\
\text { Médio } \\
\text { incompleto }\end{array}$ & $\begin{array}{c}\text { Ensino } \\
\text { Médio } \\
\text { completo }\end{array}$ & $\begin{array}{l}\text { Ensino } \\
\text { Superior }\end{array}$ & $\begin{array}{l}\text { Nunca } \\
\text { estudou }\end{array}$ & \\
\hline \multicolumn{8}{|c|}{ Autopercepção sobre a necessidade de tratamento odontológico } \\
\hline \multirow{2}{*}{ Sim } & 17 & 6 & 7 & 25 & 1 & 1 & 0,499 \\
\hline & $89,5 \%$ & $100,0 \%$ & $100,0 \%$ & $100,0 \%$ & $100,0 \%$ & $100,0 \%$ & \\
\hline \multirow{2}{*}{ Não } & 2 & 0 & 0 & 0 & 0 & 0 & \\
\hline & $10,5 \%$ & $0,0 \%$ & $0,0 \%$ & $0,0 \%$ & $0,0 \%$ & $0,0 \%$ & \\
\hline \multicolumn{8}{|c|}{ Realiza acompanhamento com o cirurgião-dentista } \\
\hline \multirow{2}{*}{ Sim } & 10 & 4 & 1 & 10 & 1 & 0 & 0,266 \\
\hline & $50,0 \%$ & $66,7 \%$ & $14,3 \%$ & $38,5 \%$ & $100,0 \%$ & $0,0 \%$ & \\
\hline \multirow{2}{*}{ Não } & 10 & 2 & 6 & 16 & 0 & 1 & \\
\hline & $50,0 \%$ & $33,3 \%$ & $85,7 \%$ & $61,5 \%$ & $0,0 \%$ & $100,0 \%$ & \\
\hline \multicolumn{8}{|c|}{ Foi orientada sobre a saúde bucal } \\
\hline \multirow{2}{*}{ Sim } & 10 & 5 & 3 & 13 & 1 & 0 & 0,465 \\
\hline & $52,6 \%$ & $83,3 \%$ & $42,9 \%$ & $50,0 \%$ & $100,0 \%$ & $0,0 \%$ & \\
\hline \multirow{2}{*}{ Não } & 9 & 1 & 4 & 13 & 0 & 1 & \\
\hline & $47,4 \%$ & $16,7 \%$ & $57,1 \%$ & $50,0 \%$ & $0,0 \%$ & $100,0 \%$ & \\
\hline \multicolumn{8}{|c|}{ Existe procedimento odontológico que não pode ser realizado durante a gestação } \\
\hline \multirow{2}{*}{ Sim } & 9 & 1 & 1 & 9 & 1 & 0 & 0,493 \\
\hline & $47,4 \%$ & $20,0 \%$ & $20,0 \%$ & $45,0 \%$ & $100,0 \%$ & $0,0 \%$ & \\
\hline \multirow{2}{*}{ Não } & 10 & 4 & 4 & 11 & 0 & 1 & \\
\hline & $52,6 \%$ & $80,0 \%$ & $80,0 \%$ & $55,0 \%$ & $0,0 \%$ & $100,0 \%$ & \\
\hline
\end{tabular}

* $p<0,05$, teste qui-quadrado ou exato de Fisher. Dados expressos em forma de frequência absoluta e percentual.

Fonte: Elaborada pelas autoras.

Ao realizar a correlação entre os dados encontrados, não se observou relação estatística entre a escolaridade das mães e as demais variáveis, tendo sido encontrados os seguintes valores $p$ : autopercepção sobre o atendimento odontológico durante o pré-natal $(p=0,499)$, acompanhamento com o cirurgião-dentista ( $p=$ $0,266)$, recebimento de orientação sobre a saúde bucal $(p=0,465)$ e procedimentos odontológicos que não podem ser executados durante a gestação $(p=0,493)$.

Quanto à avaliação dos dados qualitativos, pôde-se categorizar as falas das gestantes em: a) acesso ao serviço odontológico; e b) influência da saúde bucal na gestação.

$\mathrm{Na}$ categoria acesso ao serviço odontológico, as gestantes demonstraram saber da importância de ir ao dentista, porém, relataram dificuldades no acesso ao serviço odontológico:

[...] sei que era bom que fosse assim, que a gente tivesse a mesma facilidade como eu tô tendo agora gestante. Fosse assim em outra época também, porque eu vi mais... prioridades agora na gestação, entendeu? Porque dentista aqui é muito difícil. (Gestante 1)

A categoria influência da saúde bucal na gestação traz a discussão da importância do acompanhamento odontológico, com o objetivo de evitar complicações durante a gravidez, e evidencia a necessidade de informar mais claramente as gestantes sobre esse assunto: 
[...] eu fiquei sabendo, né, de alguns detalhes que influenciam, né?! Eu fico meio assim, na dúvida ainda, porque diz que cárie causa aborto, não sei. Ou é tártaro que causa aborto, não sei o quê, fiquei sabendo disso agora recentemente nessa gravidez, nem sabia desse assunto. (Gestante 2) Acho, sim... que às vezes se você num... Num acompanhar pelo dentista, pode você ter alguma bactéria, que pode passar para a criança, né... Tenho muito medo também... Por causa disso também. Tanto é que eu indico muito a minha filha, escovo muito o dente dela, para evitar... mas que eu sei que ela vai crescer, né... (Gestante 3)

É importante porque... grávida enjoa muito, e tem ânsia de vômito e fica tipo com uns resíduos nos dentes. Então, é bom, tanto para poder não prejudicar a criança, né. (Gestante 4)

\section{DISCUSSÃO}

Atualmente, a incidência de gravidez na adolescência é alta, estando associada a baixo nível socioeconômico, início de vida sexual precoce e questões sociais e familiares, sendo considerada um problema de saúde pública que pode acarretar agravos emocionais, familiares e sociais. Muitas vezes, essas gestantes param de estudar para cuidar de seus filhos, o que também pode resultar em diminuição de sua autoestima ${ }^{14}$. Contudo, nesta pesquisa, observou-se prevalência de mulheres em idade adulta, demonstrando que, com o amadurecimento, as mulheres tendem a procurar com maior frequência os serviços de saúde, comparecendo às consultas pré-natal de modo mais efetivo.

0 atendimento odontológico das gestantes ainda se mostra um desafio, tanto para as gestantes quanto para os profissionais da saúde. Essas mulheres fazem parte de um grupo especial e prioritário para o atendimento odontológico no Sistema Único de Saúde (SUS) devido às alterações fisiológicas e psicossociais. Contudo, a falta de interesse, a falta de orientação e o medo de ir ao dentista podem induzir a gestante a procurar atendimento apenas quando já está com um quadro de saúde bucal precário ${ }^{7,15}$. Observou-se que existe boa compreensão acerca da necessidade de tratamento odontológico, contudo, apenas $42,6 \%$ das gestantes realizam tratamento odontológico.

\section{0 atendimento odontológico das gestantes ainda se mostra um desafio...}

Também se destaca a insegurança no atendimento às gestantes por parte dos profissionais da saúde, que, muitas vezes, sobrepõe-se ao atendimento não prioritário, postergando e comprometendo esse acompanhamento, pois a demora no atendimento favorece o surgimento e agravamento de doenças bucais, que podem vir a causar danos maiores às futuras mães e aos nascituros ${ }^{7}$.

Ressalta-se que, além de realizar o procedimento clínico, trabalhar a promoção da saúde junto às gestantes assume vital importância. Isso porque se acredita que estar bem informada e motivada para cuidar de sua saúde bucal é um passo crucial para a prevenção de doenças bucais tanto na mulher como em seu filho, favorecendo a adoção de hábitos de higiene bucal saudáveis ${ }^{9}$.

0 estudo em questão observou que $52,5 \%$ das gestantes foram orientadas sobre os cuidados com sua saúde bucal, demonstrando uma necessidade das equipes da Estratégia Saúde da Família (ESF) trabalharem de modo mais incisivo a promoção da saúde, orientando acerca dos cuidados com a saúde bucal e demonstrando quais benefícios serão alcançados com essas atividades.

Deve-se ressaltar que durante a gravidez ocorrem diversas mudanças fisiológicas, psicológicas e sociais que predispõem a riscos de saúde bucal ${ }^{16-17}$. Contudo, $70 \%$ das gestantes acreditam não haver alterações na saúde bucal ao longo do período gestacional.

As evidências mostram que algumas alterações podem ocorrer com maior frequência, principalmente no periodonto, que é responsável pela sustentação e proteção do dente, devido a deficiências nutricionais, altos níveis de estrógeno e progesterona, presença de placa bacteriana e o estado transitório de imunodepressão,12,16-17. Tais fatores ocasionam 0 desenvolvimento de doenças, principalmente a doença periodontal, que se relaciona ao nascimento de bebês prematuros e de baixo peso ${ }^{16-18}$.

A influência da saúde bucal na gestação é 
compreendida pela maioria das participantes $(72,1 \%)$. Diante disso, ressalta-se que a prematuridade representa um problema econômico e de saúde pública com grande impacto social; o processo fisiológico que desencadeia o início do trabalho de parto ainda é desconhecido, mas se acredita que tenha 2 vias: a) a indireta, onde os tecidos periodontais inflamados passariam a atuar como um reservatório de bactérias, as quais se deslocariam via corrente sanguínea até a cavidade uterina; e b) a direta, onde os próprios sítios periodontais produziriam mediadores inflamatórios e estes passariam a atuar como potenciais fontes de citocinas fetotóxicas ${ }^{15-16,18}$.

Quanto aos cuidados durante o tratamento odontológico, destaca-se que é considerado seguro o uso de anestésicos em dosagem terapêutica adequada para cada paciente ${ }^{9}$. 0 mais utilizado em pacientes nessa condição é a lidocaína a $2 \%$ com adrenalina, com dose limite de no máximo 2 tubetes por sessão ${ }^{19-20}$. 0 s exames radiográficos são um procedimento seguro para gestantes, devido à quantidade de raios- $X$ e ao tempo de exposição serem pequenos, bem como devido ao fato da radiação se restringir à área exposta. 0 exame radiográfico pode ser realizado durante todo o pré-natal, quando se mostrar necessário, devendo-se recorrer a todos os métodos de proteção, como filme ultrarrápido, diafragma, filtro de alumínio, localizador, avental de chumbo e protetor de tireoide de chumbo $0^{7,13,19}$.

As cirurgias podem ser realizadas, de preferência no segundo trimestre da gestação; devido à redução dos sintomas da gravidez, não se deve realizar um atendimento prolongado. 0 tratamento endodôntico também pode ser realizado, sendo mais indicado no segundo trimestre do período gestacional ${ }^{19}$.

\section{CONCLUSÃO}

As gestantes reconhecem a importância do tratamento odontológico no período pré-natal, porém, o acompanhamento com o cirurgião-dentista ainda não é uma rotina nos serviços de saúde investigados. Verificou-se dificuldade de acesso aos serviços odontológicos, mesmo para as gestantes, um grupo de atendimento prioritário, devido às repercussões que a doença periodontal pode trazer para as mães e os bebês.

A maioria das entrevistadas acredita não haver alterações na saúde bucal das gestantes, contudo, elas compreendem que a saúde bucal tem influência na gestação. Deve-se considerar como limitação o

\section{...o acompanhamento com o cirurgião-dentista ainda não é uma rotina nos serviços de saúde investigados...}

fato desta pesquisa enfocar uma regional específica de Fortaleza, assim, recomenda-se uma ampliação da busca para as demais regionais desse município, de modo a proporcionar uma investigação mais abrangente no contexto de uma metrópole do Nordeste brasileiro.

Conclui-se que a gestação é cercada por mitos e dúvidas que dificultam o atendimento odontológico durante o pré-natal. Portanto, trabalhar a promoção de saúde com esse grupo ajuda a desmistificar tal acompanhamento, bem como a prevenir o desenvolvimento de doenças bucais, proporcionando melhor qualidade de vida tanto à futura mãe quanto ao nascituro.

\section{CONTRIBUIÇÃO DAS AUTORAS}

Diana Larissa Leitão Botelho e Vivian Gomes Azevedo Lima contribuíram com a realização da pesquisa, o delineamento do estudo e a redação do manuscrito. Myrna Maria Arcanjo Frota Barros contribuiu com o delineamento do estudo e a redação do manuscrito. Janaina Rocha de Sousa Almeida contribuiu com o delineamento do estudo e a revisão crítica do manuscrito.

\section{REFERÊNCIAS}

1. Codata LAB, Nakama L, Cordoni Júnior L, Higasi MS. Atenção odontológica à gestante: papel dos profissionais de saúde. Ciênc Saúde Colet [serial on the internet]. 2011 [cited 2019 Dec 16];16(4):2297301. Available from: http://www.scielo.br/pdf/csc/ v16n4/v16n4a29.pdf

2. Cabral MCB, Santos TS, Moreira TP. Percepção das gestantes do Programa de Saúde da Família em relação à saúde bucal no município de Ribeirópolis, Sergipe, Brasil. Rev Port Saúde Pública [serial on the internet]. 2013 [cited 2019 Dec 16];31(2):173-80. Available from: http://www.scielo.mec.pt/pdf/rpsp/ v31n2/v31n2a06.pdf 
3. Kloetezel MK, Huebner CE, Milorom P. Referrals for dental care during pregnancy. J Midwifery Womens Health [serial on the internet]. 2011 [cited 2019 Dec 16];56(2):110-7. Available from: https://www.ncbi. nlm.nih.gov/pmc/articles/PMC3074205/

4. Praetzel JR, Ferreira FV, Lenzi TL. Percepção materna sobre atenção odontológica e fonoaudiológica na gravidez. Rev Gaúch Odontol [serial on the internet]. 2010 [cited 2019 Dec 16];58(2):155-6. Available from: file:///D:/RG02007-864.pdf

5. Catão CDS, Gomes TA, Rodrigues RQF, Soares RSC. Evaluation of the knowledge of pregnant women about the relationship between oral diseases and pregnancy complications. Rev 0dontol UNESP [serial on the internet]. 2015 [cited 2019 Dec 16];44(1):5965. Available from: http://www.scielo.br/pdf/ rounesp/v44n1/1807-2577-rounesp-44-01-00059.pdf

6. Faquim JPS, Frazão P. Percepções e atitudes sobre relações interprofissionais na assistência odontológica durante o pré-natal. Saúde Debate [serial on the internet]. 2016 [cited 2019 Dec 16];40(109):59-69. Available from: https:// www.scielosp.org/scielo.php?pid =S010311042016000200059 \&script $=$ sci arttext

7. Nascimento EP, Andrade FS, Costas AMDD, Terra FS. Gestantes frente ao tratamento odontológico. Rev Bras 0dontol [serial on the internet]. 2012 [cited 2019 Dec 16];69(1):125-30. Available from: http:// revodonto.bvsalud.org/pdf/rbo/v69n1/a28v69n1.pdf

8. Santos Neto ET, Oliveira AE, Zandonade E, Leal MC. Acesso à assistência odontológica no acompanhamento pré-natal. Ciênc Saúde Colet [serial on the internet]. 2012 [cited 2019 Dec 16];17(11):3057-68. Available from: http://www. scielo.br/pdf/csc/v17n11/v17n11a21.pdf

9. Vasconcelos RG, Vasconcelos MG, Mafra RP, Queiroz LMG, Barbosa CAG. Atendimento odontológico a pacientes gestantes: como proceder com segurança. Rev Bras 0dontol [serial on the internet]. 2012 [cited 2019 Dec 16];69(1):120-4. Available from: http:// revodonto.bvsalud.org/pdf/rbo/v69n1/a27v69n1.pdf

10. Brasil. Atenção ao pré-natal de baixo risco. Brasília (DF): Ministério da Saúde; 2012. (Cadernos de Atenção Básica n. 32).

11. Reis FG, Almeida JRS. Percepção das gestantes sobre o pré-natal odontológico. Ciênc Saúde Colet. $2013 ; 4(1): 56-62$.

12. Washington DC. Dental $x$ - rays, teeth cleanings = safe during pregnancy [document on the internet]. 2013 [cited 2019 Dec 16]. Available from: https:// www.perioimplantadvisory.com/dental-implants/ hygiene-techniques/article/16411810/dental-xraysteeth-cleanings-safe-during-pregnancy
13. Alves LG, Alves LG, Pereira UX, Rocha SP, Ribeiro Junior $H$, Nepomuceno FWAB. Perfil socioeconômico de adolescentes grávidas atendidas no centro de referência da saúde da mulher na cidade de São Francisco do Conde - BA. Rev Ciênc Méd Biol [serial on the internet]. 2015 [cited 2019 Dec 16];14(2):1436. Available from: https://portalseer.ufba.br/index. php/cmbio/article/view/11791

14. Mascarenhas VI, Vilarinho LAL, Moura LFAD, Moura MS, Ferro LB. Correlação entre saúde periodontal e idade gestacional. Rev Odontol UNESP [serial on the internet]. 2012 [cited 2019 Dec 16];41(6):408-14. Available from: http://www.scielo.br/pdf/rounesp/ v41n6/a07v41n6.pdf

15. Pires BT, Alves CC, Oliveira EN, Teixeira MA. Grupo de gestante: relato de experiência. Sanare (Sobral, Online) [serial on the internet]. 2015 [cited 2019 Dec 16];14(Suppl 2):123-5. Available from: file:/// D:/883-1946-1-SM\%20(1).pdf

16. Polysos NP, Polysos IP, Zavos A, Valachis A, Mauri $D$, Papanikolaous, EG, et al. Obstetric outcomes after treatment of periodontal disease during pregnancy: systematic review and meta-analysis. BMJ. $2010 ;(341): c 7017$.

17. Marin C, Maçaneiro CA, Blittan CR, Vavarassari F. Percepção do atendimento odontológico: comparações entre grupos de gestantes adultas e adolescentes. Revista de Atenção à Saúde [serial on the internet]. 2015 [cited 2019 Dec 16];13(46):6571. Available from: https://seer.uscs.edu.br/index. php/revista ciencias saude/article/view/3099/pdf

18. Canepelle TMF, Yamamoto EC, Sousa AC. Conhecimento do cirurgião-dentista sobre 0 atendimento a pacientes especiais: hipertensos diabéticos e gestantes. Rev 0dontologia. 2011;1:3141.

19. Ibhawoh L, Enabulele, J. Endodontic treatment of the pregnant patient: knowledge, attitude and practices of dental residents. Niger Med J. 2015;56(5):311-6.

20. Martins L0, Pinheiro RDPS, Arantes DC, Nascimento LS, Santos Junior PB. Assistência odontológica à gestante: percepção do cirurgião-dentista. Revista Pan-Amazônica de Saúde [serial on the internet]. 2013 [cited 2019 Dec 16];4(4):11-8. Available from: http://scielo.iec.gov.br/pdf/rpas/v4n4/v4n4a02.pdf

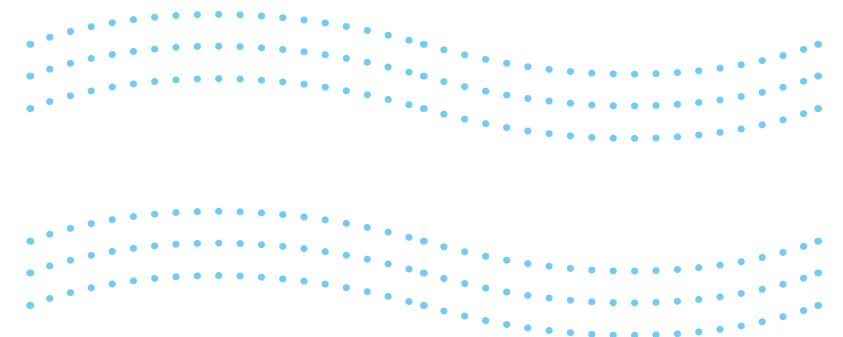

SANARE (Sobral, Online). 2019 Jul-Dec;18(2):69-77 - 77 